\title{
3-D Electron Tomographic Analysis of Mitochondria and Lipid Droplets Interactions in Heart Muscle
}

\author{
Ru-ching Hsia ${ }^{1,2}$, Tsengming Chou $^{3}$ and Carole Sztalryd-Woodle ${ }^{4}$ \\ 1. Electron Microscopy Core Imaging Facility, University of Maryland Baltimore, Baltimore, USA. \\ 2. Department of Neural \& Pain Sciences, University of Maryland School of Dentistry, Baltimore, USA. \\ 3. Laboratory for Multiscale Imaging, Stevens Institute of Technology, Hoboken, USA. \\ 4. Department of Medicine, University of Maryland Baltimore, Baltimore, USA.
}

Lipid droplets (LDs) are dynamic organelles that interact with various cell components such as the endoplasmic reticulum, endosomes and mitochondria. Excessive or defective LD accumulation are implicated in obesity and other metabolic diseases such as diabetes and atherosclerosis [1]. We describe here our investigation of the role of LDs in obesity and their interactions with other organelles using transgenic mice over-expressing perilipin 5 under a $\alpha$-myosin heavy chain promoter [2].

Due to the small size of cardiac LDs ( $\sim 1$ micron), transmission electron microscopy (TEM) is traditionally used to study LDs in heart muscle. Figure 1 illustrates ultrastructural differences in the wild type and the mutant transgenic heart muscle by traditional TEM. In normal heart muscle (Fig. 1A), mitochondria are organized between muscle fibers. In the mutant specimen, abundant LDs are present and closely associated with mitochondria in the mutant heart muscle. Furthermore, vacuoles (Fig. 1, red arrow) were seen inside the mitochondria in the LD-rich heart muscle. It is not clear what the origin and function(s) of these vacuoles are, although it has been reported that during apoptosis, the mitochondrial inner membrane may remodel and form vesicular matrix [3].

In order to further study the interspatial relationship among mitochondria, LDs, mitochondrial vesicles, endoplasmic reticulum (sarcoplasmic reticulum) and muscle fibrils in the mouse cardiac muscle, we performed scanning electron microscopy (SEM)-tomography analysis using a Zeiss Auriga FIB/SEM following a previously published protocol [4]. Two 3-D volume datasets consisting of $\sim 250$ slices of back scattered electron (BSE) SEM images were generated using focused ion beam (FIB) millings at 1nA and 240pA respectively. Figure 2 shows representative BSE images in each volume dataset. The pixel size of the dataset collected with FIB at $1 \mathrm{nA}$ is $22 \mathrm{~nm} \times 22 \mathrm{~nm} \times 20 \mathrm{~nm}$ (x, y, z) (Fig. 2A) and the pixel size for the dataset collected with FIB at 240pA is $7 \mathrm{~nm} \times 7 \mathrm{~nm} \times 10 \mathrm{~nm}$ (Fig. 2B). Higher resolution images were required to better visualize the sarcoplasmic reticulum. Image alignment, segmentation and 3D analyses were done by using ImageJ and Avizo 9.0 software.

Figure 3 illustrates a partially segmented volume of $5 \times 5 \times 5 \mu \mathrm{m}^{3}$ of mutant heart muscle. For ease of visualization, only one mitochondrion is segmented in this volume. It is clear that the integrity of mitochondrion is severely altered by the space occupied by LDs resulting in hollowed mitochondria and mitochondrial segments pinching off from the otherwise rounded mitochondrial body. Furthermore, the vacuoles (green) all originate between the mitochondrial inner membrane stacks and always appear to be adjacent to a LD (Fig. 3C). It was previously reported that one of the LD specific protein perilipin 5 promotes association of mitochondria to LDs [2]. Our 3-D tomography analyses supports the membrane interaction between LDs and mitochondria [5].

References: 
[1] M Duchen and G Szabadkai, Essays Biochem. 47 (2010), p.115.

[2] H Wang et al, Journal of Lipid Research 52 (2011), p. 2159.

[3] M Sun, Nature Cell Biology 9 (2007), p. 1557.

[4] D Wei et al, Biotechniques 53 (2012), p. 41.

[5] This research effort used microscope resources partially funded by the National Science Foundation through NSF Grant DMR-0922522.
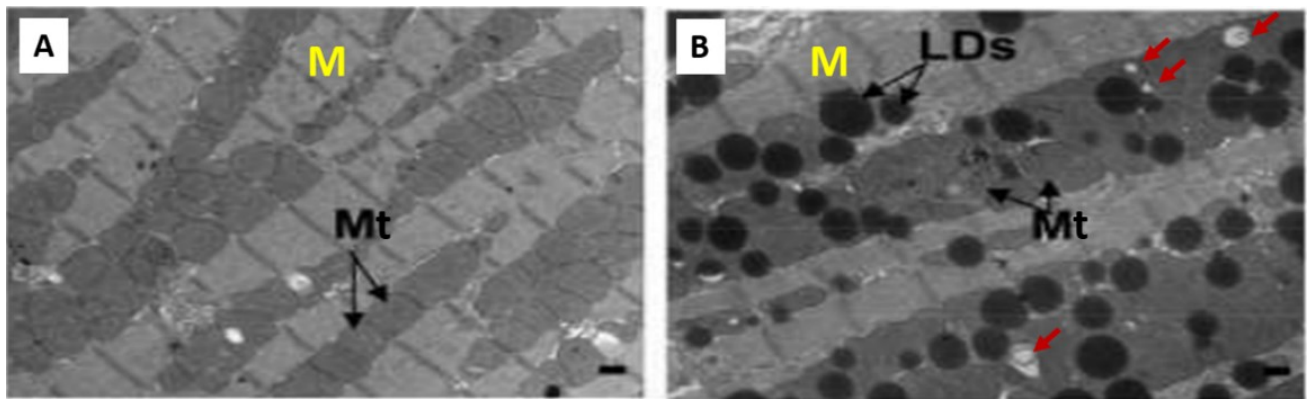

Figure 1. TEM images of murine heart muscle from a wild type (A) and transgenic mice over expressing LDs (B).
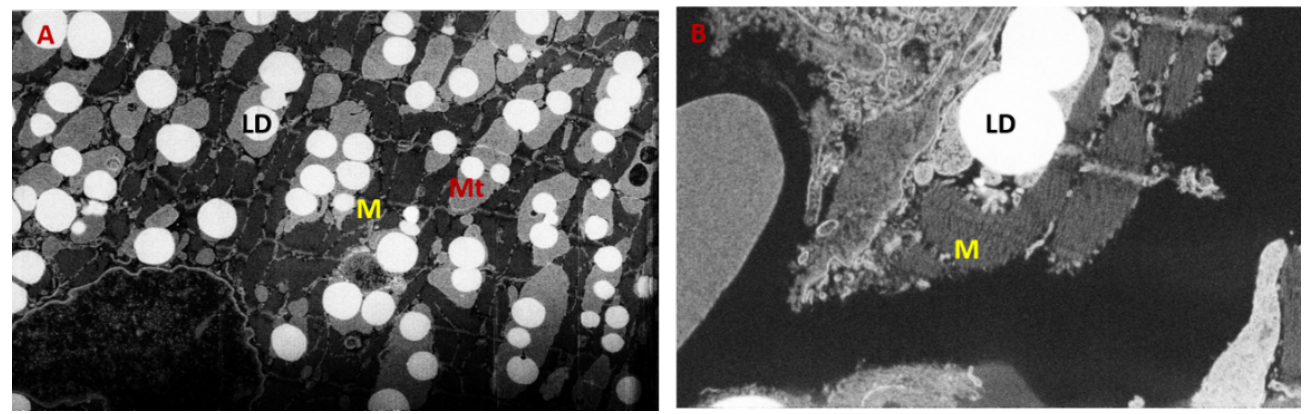

Figure 2. Representative SEM images collected using BSE detector for two separate SEM tomography experiments using FIB probe current at 1nA (A) and 240pA (B). LD: Lipid droplet. M: muscle fiber. Mt: mitochondria.
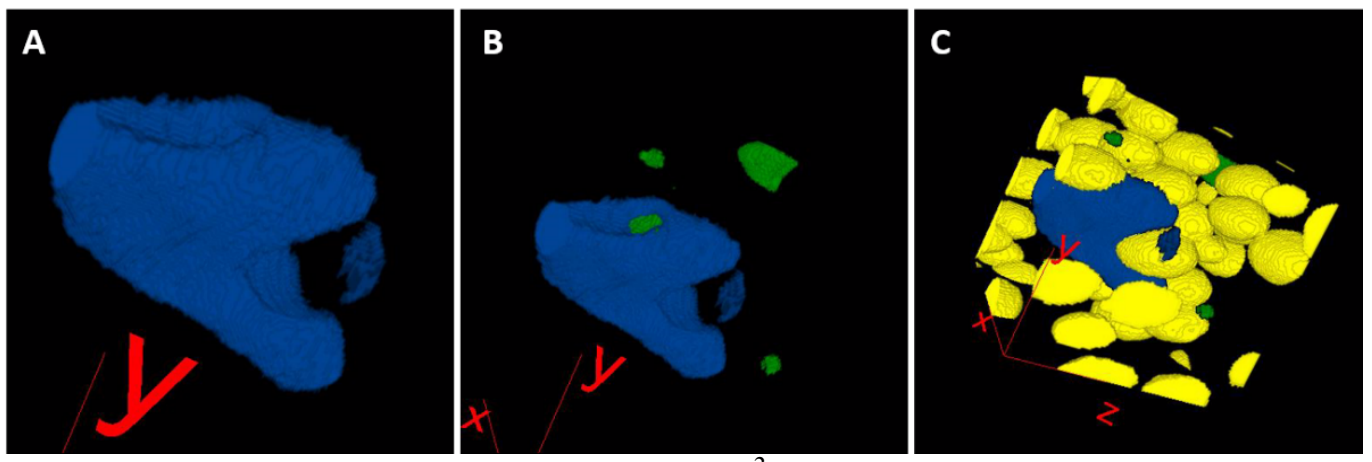

Figure 3. Segmentation and 3-D view of $\sim 5 \times 5 \times 5 \mu \mathrm{m}^{3}$ volume of mutant heart muscle overexpressing LDs. A single mitochondrion (A; blue), several mitochondrial vacuoles (B; green) and LDs (C; yellow) are shown in three successive combined images. 